\title{
La thrombocythémie héréditaire est due à une mutation du gène de la thrombopoïétine
}

La thrombocythémie familiale à transmission autosomique dominante, comme la thrombocythémie essentielle de caractère sporadique, se caractérise par une active mégacaryocytopoïèse médullaire, un nombre élevé de plaquettes circulantes, des épisodes thrombotiques et hémorragiques, et d'occasionnelles transformations blastiques. Les anomalies moléculaires de ces syndromes myéloprolifératifs sont inconnues. Radek Skoda et al. de l'université de Bâle (Suisse) rapportent dans Nature Genetics [1] qu'une mutation ponctuelle localisée dans le gène codant pour la thrombopoiétine (TPO) est la cause de la thrombocythémie héréditaire. L'étude porte sur une famille danoise dont 11 membres sur 14 sont atteints. Le premier indice suggérant une anomalie de production de TPO est l'observation que, bien que le chiffre de plaquettes soit élevé chez ces patients, les concentrations de TPO sériques décelables par ELISA sont aussi anormalement élevées. Les auteurs ont séquencé les 7 exons (2 non codants et 5 codants) [2] ainsi que les jonctions intron-exon du gène de la TPO chez un individu malade. L'analyse de la séquence révèle l'existence d'une mutation ponctuelle (transversion d'un G en $\mathrm{C}$ ) localisée dans le site donneur d'épissage de l'intron 3. Cette anomalie engendre aussi un RFLP (restriction fragment length polymorphism) qui confirme la mutation chez tous les membres atteints de la famille. La TPO étant produite essentiellement par le foie et le rein, les conséquences de la mutation sur l'épissage de l'ARNm (élimination de l'exon 3 ou rétention de l'intron 3) sont difficilement examinables. Pour résoudre ce problème, les auteurs ont utilisé une stratégie in vitro en transfectant des clones cosmidiques contenant l'ADN correspondant à l'allèle muté du gène de la TPO dans une lignée d'hépatome de rat. L'analyse effectuée par RT-PCR avec des amorces amplifiant spécifiquement les transcrits de la TPO humaine révèle les deux types de transcrits, ceux correspondant à des $\mathrm{ARNm}$ délétés de l'exon $3(\Delta \mathrm{E} 3)$ et ceux n'ayant pas épissé l'intron 3 (I3-ret), avec une prépondérance de $\Delta \mathrm{E} 3$. Dans les deux situations, l'extrémité $5^{\prime}$ des ARNm est fortement modifiée, soit par un changement complet de la séquence du signal peptide $(\Delta \mathrm{E} 3)$, soit par l'introduction d'un changement du cadre de lecture qui décale d'un exon le début de la traduction (I3-ret). Une série de constructions a été réalisée qui reproduisent les différentes possibilités d'épissage. Après transfection dans des cellules COS et mesure de l'activité biologique de la TPO dans le surnageant des cultures, les auteurs montrent que la TPO produite à partir de la forme $\Delta \mathrm{E} 3$ est sécrétée de manière beaucoup plus efficace que la TPO sauvage, tandis que la production de protéine traduite à partir des transcrits I3-ret est extrêmement faible. Deux hypothèses pouvaient expliquer l'augmentation de production de la TPO codée par l'allèle muté $\Delta \mathrm{E} 3$ : soit l'élimination de l'exon 3 pouvait augmenter l'efficacité de traduction de la protéine, soit le changement de la séquence du peptide signal pouvait améliorer la sécrétion de la protéine. Pour savoir laquelle de ces deux hypothèses était correcte, les auteurs ont étudié par transcription suivie d'une traduction in vitro en lysat de réticulocytes les différents variants de l'ARNm de la TPO et de plusieurs autres constructions dans lesquelles la longueur de la région 5' non traduite a été modifiée. Les auteurs montrent que la modification de la séquence du peptide signal ne joue pas de rôle prépondérant dans la surproduction de TPO, mais que la région 5' non traduite de l'allèle normal a un rôle inhibiteur sur l'efficacité de traduction de la protéine. En conclusion, cet élégant travail apporte pour la première fois une base moléculaire expliquant la thrombocythémie observée dans cette famille. Dans cette affection, une mutation ponctuelle affectant principalement la région 5' non traduite de la TPO conduit à une plus grande efficacité de traduction et donc à une surproduction de l'hormone. De plus, cette étude est un exemple rare montrant qu'une mutation ponctuelle dans un site donneur d'épissage peut conduire à un gain de fonction.

F.W.

1. Wiestner A, Schlemper RJ, van der Maas APC, Skoda RC. An activating splice donor mutation in the thrombopoietin gene causes hereditary thrombocytaemia. Nat Genet 1998; 18: 49-52. 2. Chang MS, McNinch J, Basu R, Shutter J, Hsu $\mathrm{RY}$, et al. Cloning and characterization of the human megakaryocyte growth and development factor (MGDF) gene. J Biol Chem 1995; 270 : 511-4. 